\title{
Perfil lipídico en mujeres embarazadas sanas de tres regiones de Chile*
}

Pablo Olmos C. ${ }^{1}$, Manuel Escalona O. 1,a , Sebastián Illanes L. ${ }^{2}$, Javier Caradeux B. ${ }^{2}$, Gabriel Mardones ${ }^{4}$, Daniela Olivari U. ${ }^{5}$, Leoncio Fuentes $V^{6}$, Ana Acosta B. ${ }^{1, a}$, Attilio Rigotti $R .{ }^{1}$, Dolores Busso P., $P h D^{1}$, José Santos M., $P h D^{1}$, José Poblete L. ${ }^{3}$, Claudio Vera P-G. ${ }^{3}$, Cristián Belmar J. ${ }^{3}$, Denisse Goldenberg A. ${ }^{1}$, Bárbara Samith ${ }^{6}{ }^{b}$, lan Niklitschek L. ${ }^{C}$, Nicolás Mertens F. $^{C}$

${ }^{1}$ Departamento de Nutrición-Diabetes y Metabolismo, Facultad de Medicina, Pontificia Universidad Católica de Chile. ${ }^{2}$ Facultad de Medicina, Universidad de Los Andes. ${ }^{3}$ División de Obstetricia-Ginecología, Facultad de Medicina, Pontificia Universidad Católica de Chile. ${ }^{4}$ Hospital Clínico San Borja Arriarán y Hospital del Profesor. ${ }^{5}$ Hospital Regional Concepción. 6Servicio de Obstetricia-Ginecología y Centro de Diagnóstico de la Mujer (CEDIM), Hospital de Talca. Chile.

${ }^{a}$ Tecnólogo-Médico. ${ }^{b}$ Nutricionista. ${ }^{c}$ Alumnos, Escuela de Medicina, Pontificia Universidad Católica de Chile.

*Proyecto de Investigación: FONDECYT N 1120682

\section{RESUMEN}

Antecedentes: Los lípidos plasmáticos maternos durante el embarazo pueden influir en el crecimiento fetal, particularmente en pacientes con diabetes gestacional; estos lípidos cambian su concentración plasmática materna a lo largo de la gestación. Objetivo: Calcular tablas y curvas de lípidos normales según edad gestacional en una población de embarazadas chilenas. Método: Se midió el colesterol total (CT), colesterol LDL (LDL-C) triglicéridos (TG), Colesterol-HDL (HDL-C), y ácidos grasos no esterificados (NEFA), en 94 embarazadas sanas y jóvenes (<33 años, edad media de 27,6 $\pm 6,2$ años), con peso pregestacional normal (Índice de Masa Corporal entre 20 y 24,9 Kg/m2 y medio de 23,3 $\pm 2,0 \mathrm{Kg} / \mathrm{m} 2$ ). Las pacientes provenían de: Hospital Parroquial de San Bernardo, Santiago $(n=55)$, Hospital de Talca $(n=2)$; Hospital del Profesor, Santiago $(n=18)$; Hospital Regional de Concepción $(n=9)$ y Hospital Clínico de la Pontificia Universidad Católica de Chile $(\mathrm{n}=10)$. Resultados: Calculamos, para cada uno de los cuatro lípidos, las curvas de percentil 50 , percentil 90 y percentil 10, en $\mathrm{mg} / \mathrm{dL}$ y mmol/l. Los NEFA solo fueron expresados en $\mathrm{mmol} / \mathrm{l}$. Incluimos las funciones matemáticas de las curvas de regresión polinomial de los cuatro lípidos con el fin que sean fácilmente reproducibles en otros tamaños. Conclusiones: Calculamos las tablas y curvas de lípidos maternos normales a lo largo del embarazo, que sean aplicables a la población de embarazadas chilenas.

PALABRAS CLAVE: Colesterol, triglicéridos, HDL, ácidos grasos, embarazo

\section{SUMMARY}

Background: In normal human pregnancy, maternal lipids can modify the rate of fetal growth, particularly in pregnancies with Gestational Diabetes Mellitus (GDM). These lipids change continuously their serum concentration in the mother along the pregnancy. Aim: To calculate tables and curves of normal serum lipids, according to gestational age, in healthy Chilean pregnant women. Methods: We measured total cholesterol (CT), LDL-cholesterol (LDL-C), triglycerides (TG), HDL-Cholesterol (HDL-C), and Non-Esterified 
Fatty Acids (NEFA) in 94 young and healthy pregnant women (<33 years, mean age $27.6 \pm 6.2$ years), with normal pregestational Body Mass Index (BMI, $20.0-24.9 \mathrm{Kg} / \mathrm{m} 2$, mean value $=23.3 \pm 2.0 \mathrm{Kg} / \mathrm{m} 2$ ). The women of the study were patients of 5 hospitals: Hospital Parroquial de San Bernardo, Santiago $(n=55)$, Hospital de Talca $(n=2)$; Hospital del Profesor, Santiago $(n=18)$; Hospital Regional de Concepción $(n=9)$ and Hospital Clínico de la Pontificia Universidad Católica de Chile $(n=10)$. Results: For each one of the lipids, we calculated curves of 50th, 90th and 10th percentiles, both in $\mathrm{mg} / \mathrm{dL}$ and $\mathrm{mmol} / \mathrm{L}$ (the NEFA were expressed only in $\mathrm{mmol} / \mathrm{L}$ ). The mathematical functions of the curves of polynomial regression of all lipids were included in the manuscript, in order to facilitate their reproduction. Conclusions: We calculated tables and curves of normal maternal serum lipids in relation to gestational, in order to make these available for use in the care of Chilean pregnant women.

\section{KEY WORDS: Cholesterol, triglycerides, HDL, fatty acids, pregnancy}

\section{INTRODUCCIÓN}

La incidencia de diabetes mellitus gestacional (DMG) ha aumentado a nivel mundial desde $4 \%$ en 1980 a 20\% en 2007 (1,2,3). La principal complicación de la DMG es la macrosomia fetal (fetos grandes para edad gestacional, GEG), que constituye un problema tanto para el obstetra como para el neonatólogo. Para el obstetra, las consecuencias son trabajo de parto prolongado, cesárea, hemorragia postparto y laceraciones vaginales (4). Para el neonatólogo, los efectos adversos incluyen fractura de clavícula, lesiones del plexo braquial, hipoglicemia, hiperbilirrubinemia, dificultad respiratoria y miocardiopatía hipertrófica $(5,6)$.

El año 2012, demostramos que la tasa de recién nacidos GEG fue de 18\%, en 251 embarazadas con DMG a pesar de un óptimo control glicémico (7). Al clasificar las madres en dos grupos según su peso pregestacional, las tasas de GEG fueron de $10 \%$ para el grupo sin sobrepeso y de $25 \%$ para las madres con sobrepeso pregestacional, a pesar que ambos grupos presentaban parámetros similares de buen control glicémico. Estos hallazgos nos demostraron que puede haber macrosomia aunque exista un adecuado control glicémico, y esto es más prevalente en el grupo de madres con sobrepeso.

En este contexto, diferentes tipos de evidencia apuntan hacia los lípidos, particularmente los triglicéridos (Figura 1), como posibles responsables del excesivo crecimiento fetal en embarazadas con DMG y sobrepeso que tienen un control glicémico óptimo (8). En otras palabras, cabe asumir que en el futuro próximo habrá un renovado interés en medir la relación entre lípidos en el suero materno y crecimiento fetal, no solo en DMG sino que en embarazadas con sobrepeso u obesidad pregestacional. Ahora bien, los lípidos en el suero materno son al menos cinco: colesterol total (CT), colesterol LDL (LDL-C), triglicéridos (TG), Colesterol-HDL
(HDL-C), y ácidos grasos no esterificados (NEFA por sus siglas en inglés, o ácidos grasos libres), y todos ellos varían considerablemente según la edad gestacional, de modo que durante el embarazo no podemos usar un solo rango como normal para cada uno de ellos. De allí entonces la necesidad de generar tablas y gráficos de percentiles normales de lípidos séricos maternos según edad gestacional en población embarazada sana y sin sobrepeso, y en lo posible, con población del país obtenida de varios centros asistenciales en más de una región geográfica.

El objetivo de esta investigación es exponer los resultados que con la cooperación de cinco Servicios de Obstetricia y Ginecología de tres regiones del país, logramos reunir para calcular las tablas y gráficos de lípidos maternos normales a lo largo del embarazo.

\section{PACIENTES Y MÉTODO}

Las pacientes (Tabla I) fueron 94 embarazadas sanas, jóvenes (rango: 20-33 años, edad media de 27,6 \pm 6,2 años), con Índice de Masa Corporal (IMC) normal pregestacional $(20-24,9 \mathrm{Kg} / \mathrm{m} 2)$. La media aritmética de IMC pregestacional fue de 23,3 $\pm 2,0 \mathrm{Kg} / \mathrm{m} 2$. Las pacientes provenían de Hospital Parroquial de San Bernardo $(n=55)$, Hospital de Talca $(n=2)$, Hospital del Profesor $(n=18)$, Hospital Regional de Concepción ( $n=9)$ y Hospital Clínico de la Pontificia Universidad Católica de Chile $(n=10)$.

Todas las pacientes fueron reclutadas bajo consentimiento informado, de acuerdo con las normas del comité de ética de cada una de las instituciones, y cumpliendo con todos los postulados de la Declaración de Helsinki.

Cada muestra de sangre fue obtenida en la mañana, luego de un ayuno nocturno de 12 horas. Una vez separado el suero, dos alícuotas fueron enviadas a los laboratorios y conservadas en refrigerador a $-80^{\circ} \mathrm{C}$ hasta su posterior análisis. 


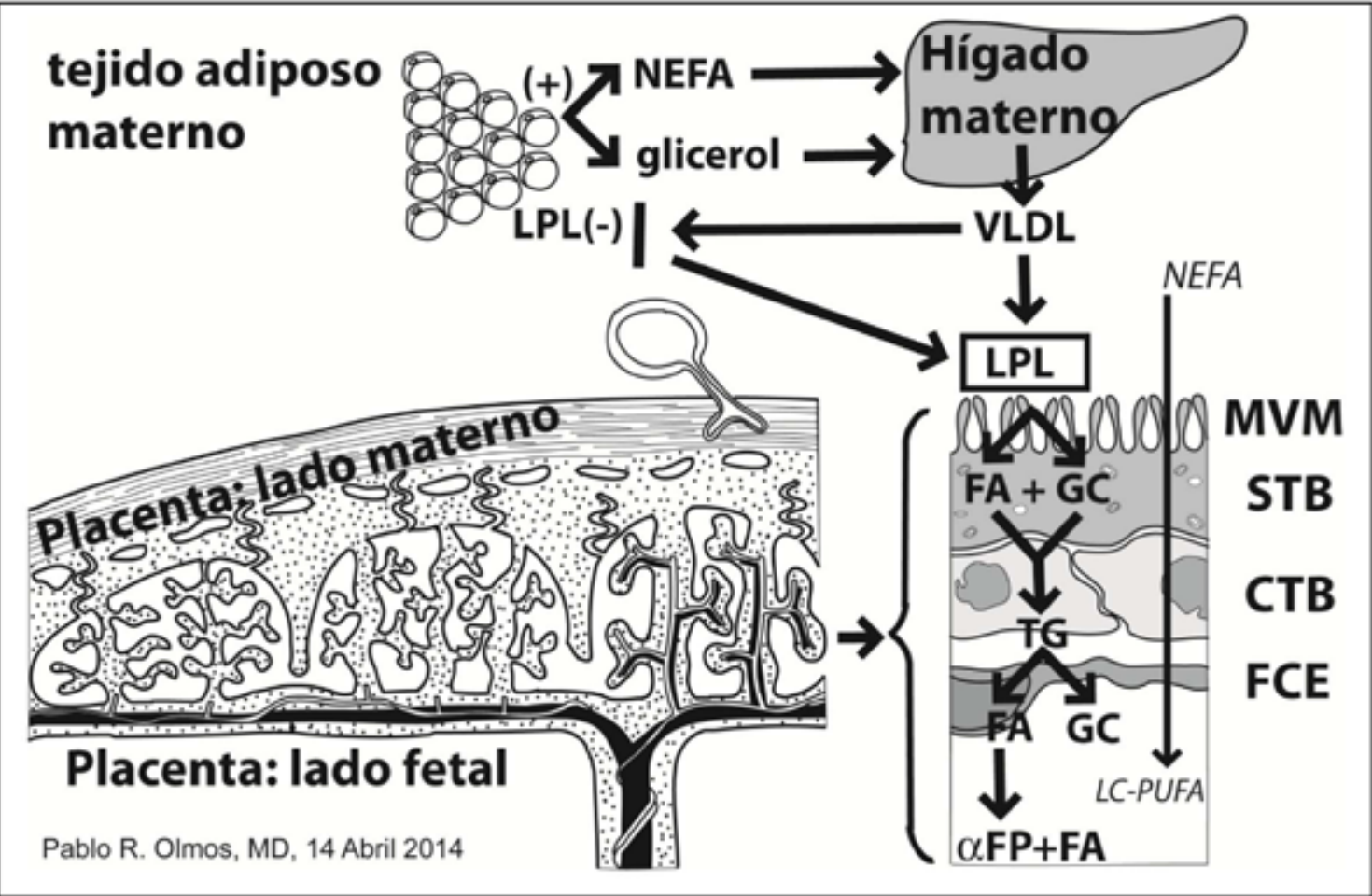

Figura 1. Transporte transplacentario de triglicéridos en un embarazo normal. NEFA= Non-Esterified Fatty Acids (Acidos Grasos No Esterificados); LPL= Liproprotein-Lipase (Lipasa Lipoproteica Hormona-Sensible); VLDL= Very Low Density Lipoprotein (Lipoproteína de muy baja densidad); FA= Fatty Acid (acido graso); $\mathrm{GC}=$ glicerol; $\mathrm{TG}=$ triglicérido; $\alpha \mathrm{FP}+\mathrm{FA}=$ ácido graso transportado por la alfa-fetoproteína. LC-PUFA= lecityn-polyunsaturated fatty acids (ácidos grasos poli-insaturados unidos a la lecitina). MVM= Membrana Vellosa Materna. STB= Sincisio-Trofoblasto. CTB= Cito-Trofoblasto. FCE= Endotelio Capilar Fetal (12-15).

Tabla I

CARACTERÍSTICAS DE LAS 94 EMBARAZADAS CHILENAS SANAS EN LAS QUE SE EFECTUÓ EL ESTUDIO

\begin{tabular}{lcccc}
\hline Procedencia & $\mathrm{n}$ & $\begin{array}{c}\text { Edad } \\
\text { gestacional } \\
\text { (semanas) }\end{array}$ & $\begin{array}{c}\text { Edad } \\
\text { cronológica } \\
\text { (años) }\end{array}$ & $\begin{array}{c}\text { IMC } \\
\text { pregestacional } \\
\text { (Kg / m2) }\end{array}$ \\
\hline Hospital Parroquial de San Bernardo & 55 & $21,6 \pm 8,9$ & $27,1 \pm 5,9$ & $22,4 \pm 1,2$ \\
Hospital de Talca & 2 & $11,0 \pm 2,8$ & $27,0 \pm 4,4$ & $22,4 \pm 1,8$ \\
Hospital del Profesor & 18 & $24,3 \pm 11,2$ & $25,8 \pm 6,7$ & $24,8 \pm 2,3$ \\
Hospital Regional de Concepción & 9 & $24,3 \pm 6,4$ & $30,2 \pm 6,5$ & $24,0 \pm 1,0$ \\
Hospital Universidad Católica & 10 & $18,9 \pm 8,4$ & $31,5 \pm 1,8$ & $24,8 \pm 3,2$ \\
\hline Totales & 94 & $21,9 \pm 9,2$ & $27,6 \pm 6,2$ & $23,3 \pm 2,0$ \\
\hline
\end{tabular}


El perfil lipídico, que incluye colesterol total (CT), colesterol LDL (LDL-C), triglicéridos (TG), Colesterol-HDL (HDL-C), fue medido con el mismo sistema en los cinco centros hospitalarios. Se utilizó muestras de suero, usando un ensayo enzimático-colorimétrico usando reactivos HUMAN (Gesellschaft für Biochemica und Diagnostica $\mathrm{mbH}$, Alemania), en autoanalizador HumaStar 80 de la misma empresa.

Los ácidos grasos no esterificados (NEFA por sus siglas en inglés, o ácidos grasos libres), fueron medidos en las muestras de suero provenientes del Hospital Parroquial de San Bernardo $(n=55)$. Se utilizaron muestras de suero, usando un ensayo colorimétrico mediante el kit NEFA Randox (Randox Laboratories Ltd., United Kingdom) procesados también en el autoanalizador HumaStar80 (Gesellschaft fbr Biochemica und Diagnostica mbH, Alemania).

Tanto los datos clínicos como los resultados de perfil lipídico y ácidos grasos libres fueron ordenados en una base de datos SPSS (PASW Statistics 18, Release 18.0.0, Julio 30th, 2009. WinWrapBasic, (C) 1993-2007, Polar Engineering and Consulting, USA). Los valores de perfil lipídico fueron guardados tanto en unidades de milígramos por decilitro $(\mathrm{mg} / \mathrm{dL})$ como de milimoles por litro $(\mathrm{mmol} / \mathrm{l})$. Los valores de ácidos grasos libres fueron archivados solamente en $\mathrm{mmol} / \mathrm{l}$.

Se obtuvieron muestras para las semanas 9, $11,13,22,24,27,33,35$ y 38 . El número de muestras para cada semana fueron, respectivamente: 9 , $15,9,21,8,6,19,4$ y 3 . Para cada una de las semanas mencionadas, se calculó la media y la desviación estándar $( \pm \mathrm{DE})$. El percentil 90 (P-90) se calculó sumando a la media la desviación estándar multiplicada por 1,282. El percentil 10 (P-10) se calculó restando a la media la desviación estándar multiplicada por 1,282 .

Una vez obtenidos las medias, DE, P-90 y P-10, aplicamos un segundo programa computacional, Prism-6 For Windows, Versión 6.04-Trial (@ 19922014 GraphPad Software Inc., USA). Para cada uno de los lípidos y para los ácidos grasos libres, obtuvimos las curvas de regresión polinomial de segundo orden de medias, DE, P-90 y P-10, las que fueron impresas en gráficos cuyo eje $X$ (abscisa) era las semanas de embarazo. Además, presentamos en una tabla las ecuaciones de las funciones polinomiales, tanto en $\mathrm{mg} / \mathrm{dL}$ como en $\mathrm{mmol} / \mathrm{l}$, con el fin que estas curvas puedan ser impresas por otros autores e investigadores en forma precisa y con el tamaño que ellos consideren adecuado a sus propósitos.

\section{RESULTADOS}

La Tabla II muestra el colesterol total materno, con sus valores medios, DE, P-90 y P-10, expresados tanto en $\mathrm{mg} / \mathrm{dL}$ como en $\mathrm{mmol} / \mathrm{l}$. La Tabla III muestra, para el colesterol total materno, las ecuaciones de regresión polinomial de segundo orden de los percentiles (50, 90 y 10). La Figura 2, muestra los percentiles P-50, P-90 y P-10 de colesterol total materno, calculados a partir de las ecuaciones polinomiales de la Tabla II.

\section{Tabla II}

COLESTEROL TOTAL MATERNO EN 94 MADRES EMBARAZADAS SANAS PROVENIENTES DE CINCO HOSPITALES EN TRES REGIONES DE CHILE, CON VALORES EXPRESADOS TANTO EN MG/DL COMO EN MMOL/L

\begin{tabular}{cccccccccc}
\hline Se & $\mathrm{n}$ & $\begin{array}{c}\mathrm{CT} \\
\mathrm{media} \\
\mathrm{mg} / \mathrm{dl}\end{array}$ & $\begin{array}{c}\mathrm{CT} \\
\pm \mathrm{DE} \\
\mathrm{mg} / \mathrm{dl}\end{array}$ & $\begin{array}{c}\mathrm{CT} \\
\mathrm{P}-90 \\
\mathrm{mg} / \mathrm{dl}\end{array}$ & $\begin{array}{c}\mathrm{CT} \\
\mathrm{P}-10 \\
\mathrm{mg} / \mathrm{dl}\end{array}$ & $\begin{array}{c}\mathrm{CT} \\
\mathrm{media} \\
\mathrm{mmol} / \mathrm{l}\end{array}$ & $\begin{array}{c}\mathrm{CT} \\
\pm \mathrm{DE} \\
\mathrm{mmol} / \mathrm{l}\end{array}$ & $\begin{array}{c}\mathrm{CT} \\
\mathrm{P}-90 \\
\mathrm{mmol} / \mathrm{I}\end{array}$ & $\begin{array}{c}\mathrm{CT} \\
\mathrm{P}-10 \\
\mathrm{mmol} / \mathrm{l}\end{array}$ \\
\hline 9 & 9 & 153,40 & 24,89 & 185,31 & 121,49 & 3,97 & 0,64 & 4,79 & 3,14 \\
11 & 15 & 198,66 & 32,80 & 240,71 & 156,61 & 5,14 & 0,85 & 6,22 & 4,05 \\
13 & 9 & 189,10 & 33,90 & 232,56 & 145,64 & 4,89 & 0,88 & 6,01 & 3,77 \\
22 & 21 & 254,20 & 48,08 & 315,84 & 192,56 & 6,57 & 1,24 & 8,17 & 4,98 \\
24 & 8 & 237,18 & 43,50 & 292,95 & 181,41 & 6,13 & 1,12 & 7,58 & 4,69 \\
27 & 6 & 249,20 & 40,22 & 300,76 & 197,64 & 6,44 & 1,04 & 7,78 & 5,11 \\
33 & 19 & 265,55 & 60,74 & 343,42 & 187,68 & 6,87 & 1,57 & 8,88 & 4,85 \\
35 & 4 & 246,75 & 17,65 & 269,38 & 224,12 & 6,38 & 0,46 & 6,97 & 5,80 \\
38 & 3 & 239,33 & 27,15 & 274,14 & 204,52 & 6,19 & 0,70 & 7,09 & 5,29 \\
\hline
\end{tabular}

$\mathrm{Se}=$ semanas de embarazo. $\mathrm{n}=$ número de pacientes. $\mathrm{CT}=$ colesterol total. Media=media aritmética (percentil 50). DE=desviación estándar. P-90=percentil 90 (media + [1,282 x DE]). P-10=percentil 10 (media - [1,282 x DE]). 
Tabla III

COLESTEROL TOTAL MATERNO: ECUACIONES DE REGRESIÓN POLINOMIAL DE SEGUNDO ORDEN PARA LOS PERCENTILES (50, 90 Y 10), QUE PERMITIERON CONSTRUIR EL GRÁFICO DE LA FIGURA 2, CON VALORES EXPRESADOS TANTO EN MG/DL COMO EN MMOL/L

\begin{tabular}{llc}
\hline & Ecuación polinomial de segundo orden & ${\text { Ecuación } \mathrm{N}^{\circ}}$ \\
\hline Percentil $50(\mathrm{mg} / \mathrm{dL})$ & $\mathrm{Y}=65,66+(12,84 \mathrm{X})-\left(0,2163 \mathrm{X}^{2}\right)$ & 1 \\
Percentil $90(\mathrm{mg} / \mathrm{dL})$ & $\mathrm{Y}=54,16+(18,85 \mathrm{X})-\left(0,3428 \mathrm{X}^{2}\right)$ & 2 \\
Percentil $10(\mathrm{mg} / \mathrm{dL})$ & $\mathrm{Y}=77,15+(6,834 \mathrm{X})-\left(0,08982 \mathrm{X}^{2}\right)$ & 3 \\
Percentil $50(\mathrm{mmol} / \mathrm{l})$ & $\mathrm{Y}=1,71+(0,3307 \mathrm{X})-\left(0,005565 \mathrm{X}^{2}\right)$ & 4 \\
Percentil $90(\mathrm{mmol} / \mathrm{l})$ & $\mathrm{Y}=1,38+(0,4885 \mathrm{X})-\left(0,008884 \mathrm{X}^{2}\right)$ & 5 \\
Percentil $10(\mathrm{mmol} / \mathrm{l})$ & $\mathrm{Y}=1,99+(0,1765 \mathrm{X})-\left(0,002317 \mathrm{X}^{2}\right)$ & 6 \\
\hline
\end{tabular}

$\mathrm{Y}=$ colesterol total (en $\mathrm{mg} / \mathrm{dL}$ para ecuaciones 1,2 , 3 y en $\mathrm{mmol} / \mathrm{L}$ para ecuaciones $4,5,6)$. $\mathrm{X}=$ semana de embarazo.

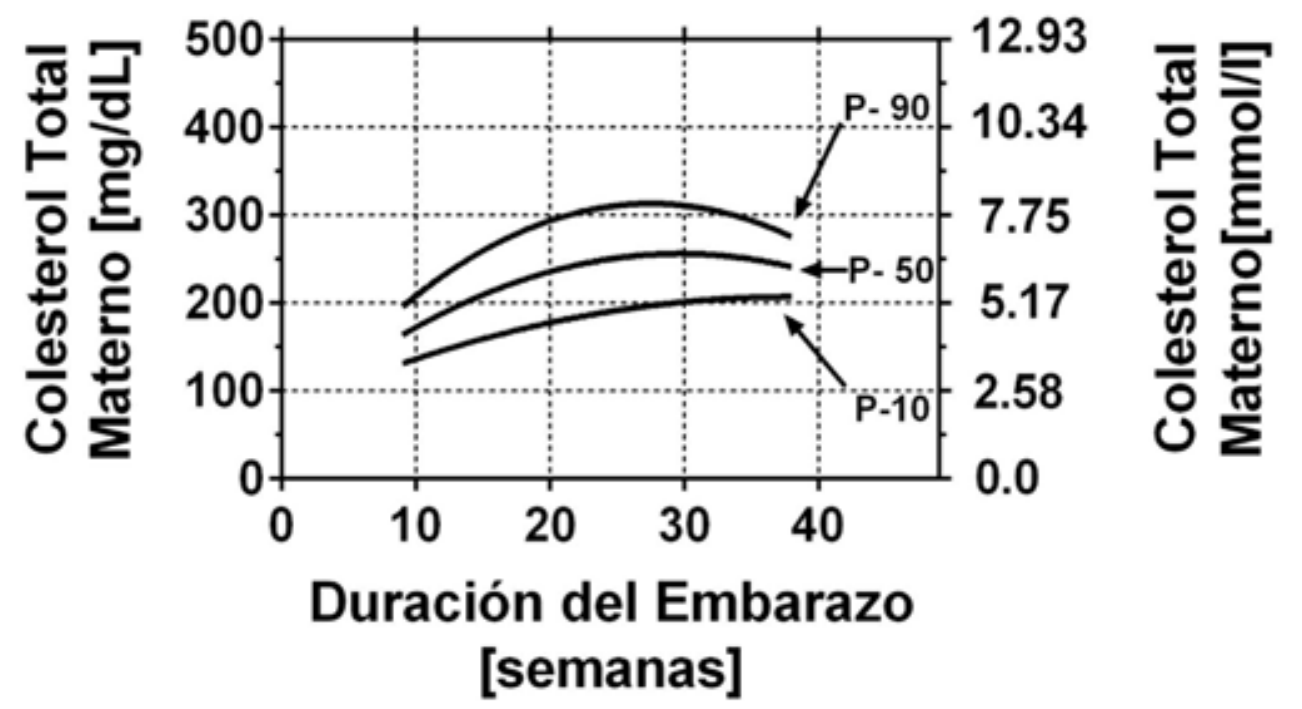

Figura 2. Colesterol total en el suero materno durante el embarazo. Percentil 50 (P-50), Percentil 90 (P-90) y Percentil 10 (P-10) calculados a partir de mujeres embarazadas sanas chilenas con IMC normal pregestacional. Valores de Colesterol Total expresados tanto en $\mathrm{mg} / \mathrm{dL}$ (eje $\mathrm{Y}$, a izquierda) como en $\mathrm{mmol} / \mathrm{l}$ (eje $\mathrm{Y}$, a derecha).

La Tabla IV muestra el colesterol LDL materno, con sus valores medios, DE, P-90 y P-10, expresados tanto en $\mathrm{mg} / \mathrm{dL}$ como en $\mathrm{mmol} / \mathrm{l}$. La Tabla $\mathrm{V}$ muestra, para el colesterol LDL materno, las ecuaciones de regresión polinomial de segundo orden de los percentiles $(50,90$ y 10$)$. La Figura 3, muestra los percentiles $\mathrm{P}-50, \mathrm{P}-90$ y $\mathrm{P}-10$ de colesterol total materno, calculados a partir de las ecuaciones polinomiales de la Tabla $\mathrm{V}$.
La Tabla VI muestra los triglicéridos maternos, con sus valores medios, DE, P-90 y P-10, expresados tanto en $\mathrm{mg} / \mathrm{dL}$ como en $\mathrm{mmol} / \mathrm{l}$. La Tabla VII muestra, para los triglicéridos maternos, las ecuaciones de regresión polinomial de segundo orden de los percentiles $(50,90$ y 10). La Figura 4, muestra los percentiles P-50, P-90 y P-10 de triglicéridos maternos, calculados a partir de las ecuaciones polinomiales de la Tabla VII. 
Tabla IV

COLESTEROL LDL MATERNO EN 94 MADRES EMBARAZADAS SANAS PROVENIENTES DE CINCO HOSPITALES EN TRES REGIONES DE CHILE, CON VALORES EXPRESADOS TANTO EN MG/DL COMO EN MMOL/L

\begin{tabular}{|c|c|c|c|c|c|c|c|c|c|}
\hline $\mathrm{Se}$ & $\mathrm{n}$ & $\begin{array}{l}\text { LDL-C } \\
\text { media } \\
\text { mg/dl }\end{array}$ & $\begin{array}{l}\text { LDL-C } \\
\pm \mathrm{DE} \\
\mathrm{mg} / \mathrm{dl}\end{array}$ & $\begin{array}{l}\text { LDL-C } \\
\mathrm{P}-90 \\
\mathrm{mg} / \mathrm{dl}\end{array}$ & $\begin{array}{l}\text { LDL-C } \\
\mathrm{P}-10 \\
\mathrm{mg} / \mathrm{dl}\end{array}$ & $\begin{array}{l}\mathrm{LDL}-\mathrm{C} \\
\mathrm{media} \\
\mathrm{mmol} / \mathrm{l}\end{array}$ & $\begin{array}{c}\text { LDL-C } \\
\pm \mathrm{DE} \\
\mathrm{mmol} / \mathrm{l}\end{array}$ & $\begin{array}{l}\text { LDL-C } \\
\text { P-90 } \\
\mathrm{mmol} / \mathrm{l}\end{array}$ & $\begin{array}{l}\text { LDL-C } \\
\text { P-10 } \\
\mathrm{mmol} / \mathrm{l}\end{array}$ \\
\hline 9 & 9 & 87,00 & 23,20 & 116,74 & 57,26 & 2,25 & 0,60 & 3,02 & 1,48 \\
\hline 11 & 15 & 103,97 & 30,50 & 143,07 & 64,87 & 2,69 & 0,79 & 3,70 & 1,68 \\
\hline 13 & 9 & 109,00 & 19,46 & 133,95 & 84,05 & 2,82 & 0,50 & 3,46 & 2,17 \\
\hline 22 & 20 & 121,53 & 34,67 & 165,98 & 77,08 & 3,14 & 0,90 & 4,29 & 1,99 \\
\hline 24 & 8 & 123,02 & 46,30 & 182,38 & 63,66 & 3,18 & 1,20 & 4,72 & 1,65 \\
\hline 27 & 6 & 155,30 & 40,01 & 206,59 & 104,01 & 4,02 & 1,03 & 5,34 & 2,69 \\
\hline 33 & 17 & 132,38 & 41,03 & 184,98 & 79,78 & 3,42 & 1,06 & 4,78 & 2,06 \\
\hline 35 & 3 & 113,86 & 32,31 & 155,28 & 72,44 & 2,94 & 0,84 & 4,02 & 1,87 \\
\hline 38 & 3 & 98,46 & 19,51 & 123,47 & 73,45 & 2,55 & 0,50 & 3,19 & 1,90 \\
\hline
\end{tabular}

$\mathrm{Se}=$ semanas de embarazo. $\mathrm{n}=$ úmero de pacientes. LDL-C=colesterol LDL. Media=media aritmética (percentil 50). $\mathrm{DE}=$ desviación estándar. P-90=percentil 90 (= media + [1,282 x DE]). P-10=percentil 10 (= media $-[1,282 \times \mathrm{DE}])$.

Tabla V

COLESTEROL LDL MATERNO: ECUACIONES DE REGRESIÓN POLINOMIAL DE SEGUNDO ORDEN PARA LOS PERCENTILES (50, $90 \mathrm{Y}$ 10), QUE PERMITIERON CONSTRUIR EL GRÁFICO DE LA FIGURA 3, CON VALORES EXPRESADOS TANTO EN MG/DL COMO EN MMOL/L

\begin{tabular}{llc}
\hline & Ecuación Polinomial de segundo orden & Ecuación N $^{\circ}$ \\
\hline Percentil $50(\mathrm{mg} / \mathrm{dL})$ & $\mathrm{Y}=19,34+(9,296 \mathrm{X})-\left(0,1859 \mathrm{X}^{2}\right)$ & 7 \\
Percentil $90(\mathrm{mg} / \mathrm{dL})$ & $\mathrm{Y}=4,652+(14,8 \mathrm{X})-\left(0,2985 \mathrm{X}^{2}\right)$ & 8 \\
Percentil $10(\mathrm{mg} / \mathrm{dL})$ & $\mathrm{Y}=34,04+(3,794 \mathrm{X})-\left(0,07335 \mathrm{X}^{2}\right)$ & 9 \\
Percentil $50(\mathrm{mmol} / \mathrm{l})$ & $\mathrm{Y}=0,5046+(0,240 \mathrm{X})-\left(0,0048 \mathrm{X}^{2}\right)$ & 10 \\
Percentil $90(\mathrm{mmol} / \mathrm{l})$ & $\mathrm{Y}=0,120+(0,3826 \mathrm{X})-\left(0,007718 \mathrm{X}^{2}\right)$ & 11 \\
Percentil $10(\mathrm{mmol} / \mathrm{l})$ & $\mathrm{Y}=0,8796+(0,09815 \mathrm{X})-\left(0,001898 \mathrm{X}^{2}\right)$ & 12 \\
\hline
\end{tabular}

$\mathrm{Y}=$ colesterol LDL (en $\mathrm{mg} / \mathrm{dL}$ para ecuaciones 7,8 , 9 y en $\mathrm{mmol} / \mathrm{L}$ para ecuaciones $10,11,12$ ). $\mathrm{X}=$ semana de embarazo.
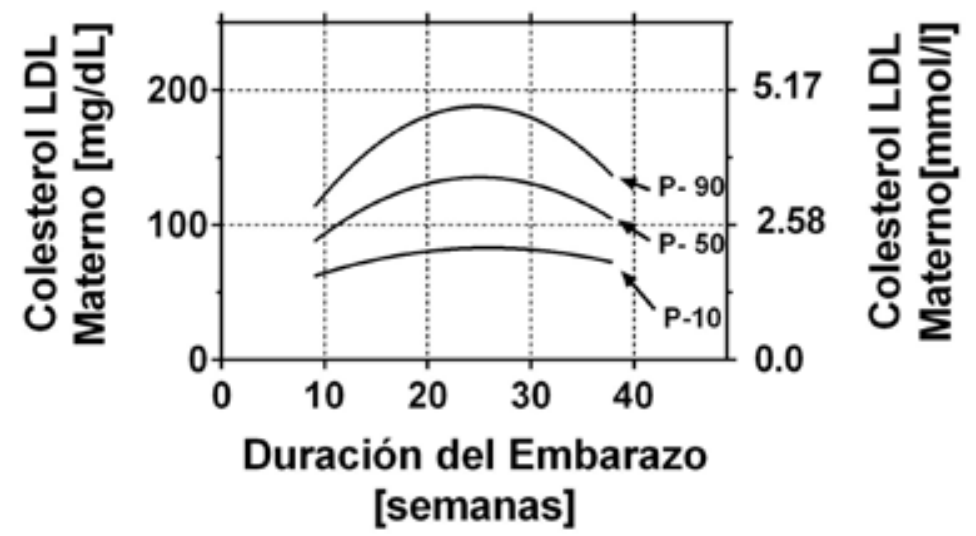

Figura 3. Colesterol LDL en el suero materno durante el embarazo. Percentil 50 (P-50), Percentil 90 (P-90) y Percentil 10 (P-10) calculados a partir de mujeres embarazadas sanas chilenas con IMC normal pregestacional. Valores de Colesterol LDL expresados tanto en $\mathrm{mg} / \mathrm{dL}$ (eje $\mathrm{Y}$, a izquierda) como en $\mathrm{mmol} / \mathrm{l}$ (eje $\mathrm{Y}$, a derecha). 


\section{Tabla VI}

TRIGLICÉRIDOS MATERNOS EN 94 EMBARAZADAS SANAS PROVENIENTES DE CINCO HOSPITALES DE TRES REGIONES DE CHILE, CON VALORES EXPRESADOS TANTO EN MG/DL COMO EN MMOL/L

\begin{tabular}{|c|c|c|c|c|c|c|c|c|c|}
\hline $\mathrm{Se}$ & $n$ & $\begin{array}{c}\mathrm{TG} \\
\text { media } \\
\mathrm{mg} / \mathrm{dl}\end{array}$ & $\begin{array}{c}\mathrm{TG} \\
\mathrm{DE} \\
\mathrm{mg} / \mathrm{dl}\end{array}$ & $\begin{array}{c}\mathrm{TG} \\
\mathrm{P}-90 \\
\mathrm{mg} / \mathrm{dl}\end{array}$ & $\begin{array}{c}\mathrm{TG} \\
\mathrm{P}-10 \\
\mathrm{mg} / \mathrm{dl}\end{array}$ & $\begin{array}{c}\text { TG } \\
\text { Media } \\
\text { mmol/l }\end{array}$ & $\begin{array}{c}\text { TG } \\
\text { DE } \\
\mathrm{mmol} / \mathrm{l}\end{array}$ & $\begin{array}{c}\text { TG } \\
\text { P-90 } \\
\mathrm{mmol} / \mathrm{l}\end{array}$ & $\begin{array}{c}\mathrm{TG} \\
\mathrm{P}-10 \\
\mathrm{mmol} / \mathrm{I}\end{array}$ \\
\hline 9 & 9 & 79,00 & 18,60 & 102,85 & 55,15 & 0,89 & 0,21 & 1,16 & 0,62 \\
\hline 11 & 15 & 130,30 & 86,25 & 240,81 & 19,79 & 1,47 & 0,97 & 2,72 & 0,22 \\
\hline 13 & 9 & 145,45 & 55,95 & 217,06 & 73,74 & 1,64 & 0,63 & 2,45 & 0,83 \\
\hline 22 & 21 & 261,65 & 69,90 & 351,21 & 171,99 & 2,95 & 0,79 & 3,97 & 1,94 \\
\hline 24 & 8 & 198,20 & 65,80 & 282,56 & 113,84 & 2,24 & 0,74 & 3,19 & 1,29 \\
\hline 27 & 6 & 192,20 & 53,35 & 260,53 & 123,87 & 2,17 & 0,60 & 2,94 & 1,40 \\
\hline 33 & 19 & 263,70 & 82,75 & 369,72 & 157,68 & 2,98 & 0,93 & 4,17 & 1,78 \\
\hline 35 & 4 & 319,50 & 63,85 & 401,29 & 237,71 & 3,61 & 0,72 & 4,53 & 2,68 \\
\hline 38 & 3 & 271,00 & 103,05 & 403,05 & 138,95 & 3,06 & 1,16 & 4,55 & 1,57 \\
\hline
\end{tabular}

$\mathrm{Se}=$ semanas de embarazo. $\mathrm{n}=$ número de pacientes. $\mathrm{TG}=$ triglicéridos maternos. Media=media aritmética (percentil 50). DE=desviación estándar. P-90=percentil 90 (=media + [1,282 x DE]). P-10=percentil 10 (= media - [1,282 x DE]).

Tabla VII

TRIGLICÉRIDOS MATERNOS: ECUACIONES DE REGRESIÓN POLINOMIAL DE SEGUNDO ORDEN PARA LOS PERCENTILES (50, 90 Y 10), QUE PERMITIERON CONSTRUIR EL GRÁFICO DE LA FIGURA 4 CON VALORES EXPRESADOS TANTO EN MG/DL COMO EN MMOL/L

\begin{tabular}{|c|c|c|}
\hline & Ecuación Polinomial de segundo orden & Ecuación $\mathrm{N}^{\circ}$ \\
\hline Percentil 50 (mg/dL) & $(-4,265)+(12,59 X)-\left(0,13 X^{2}\right)$ & 13 \\
\hline Percentil 90 (mg/dL) & $56,43+(12,78 X)-\left(0,09922 X^{2}\right)$ & 14 \\
\hline Percentil 10 (mg/dL) & $(-64,97)+(12,40 X)-\left(0,1608 X^{2}\right)$ & 15 \\
\hline Percentil 50 (mmol/l) & $Y=(-0,05121)+(0,1422 X)-\left(0,001467 X^{2}\right)$ & 16 \\
\hline Percentil 90 (mmol/l) & $Y=\quad 0,6347+(0,1446 X)-\left(0,001129 X^{2}\right)$ & 17 \\
\hline Percentil 10 (mmol/l) & $Y=(-0,7430)+(0,1408 X)-\left(0,001830 X^{2}\right)$ & 18 \\
\hline
\end{tabular}

$\mathrm{Y}=$ triglicéridos (en $\mathrm{mg} / \mathrm{dL}$ para ecuaciones 13, 14, 15 y en mmol/L para las ecuaciones 16, 17, 18). $\mathrm{X}=\mathrm{semana}$ de embarazo.
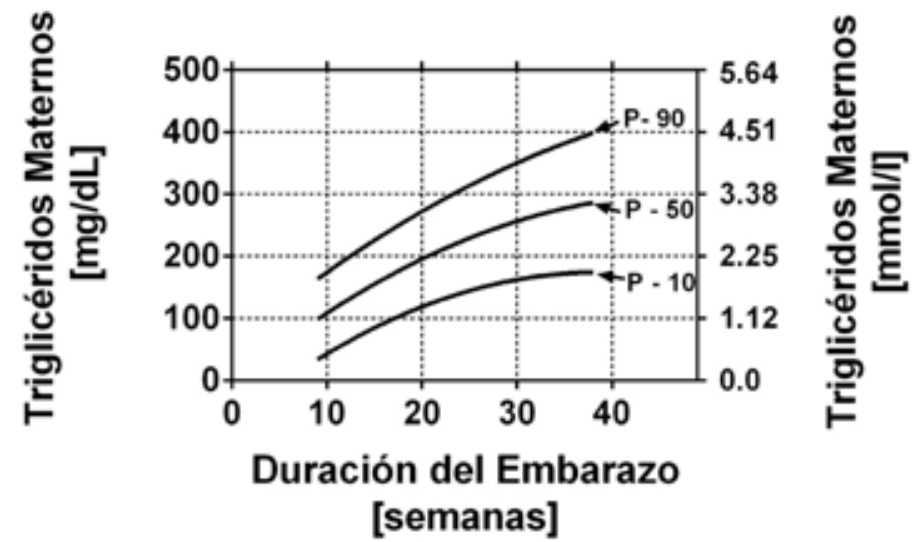

\section{[semanas]}

Figura 4: Triglicéridos en el suero materno durante el embarazo. Percentil 50 (P-50), Percentil 90 (P-90) y Percentil 10 (P-10) calculados a partir de mujeres embarazadas sanas chilenas con IMC normal pregestacional. Valores de triglicéridos expresados tanto en $\mathrm{mg} / \mathrm{dL}$ (eje $\mathrm{Y}$, a izquierda) como en $\mathrm{mmol} / \mathrm{l}$ (eje $\mathrm{Y}$, a derecha). 
La Tabla VIII muestra el colesterol HDL materno, con sus valores medios, DE, P-90 y P-10, expresados tanto en $\mathrm{mg} / \mathrm{dL}$ como en $\mathrm{mmol} / \mathrm{l}$. La Tabla IX muestra, para el colesterol HDL materno, las ecuaciones de regresión polinomial de segundo orden de los percentiles (50, 90 y 10). La Figura 5, muestra los percentiles P-50, P-90 y P-10 de colesterol HDL materno, calculados a partir de las ecuaciones polinomiales de la Tabla IX.
La Tabla $X$ muestra los NEFA maternos, con sus valores medios, DE, P-90 y P-10, expresados en $\mathrm{mmol} / \mathrm{l}$. La Tabla XI muestra, para los NEFA maternos, las ecuaciones de regresión polinomial de segundo orden de los percentiles (50,90 y 10). La Figura 6, muestra los percentiles P-50, P-90 y P-10 de NEFA maternos, calculados a partir de las ecuaciones polinomiales de la Tabla XI.

Tabla VIII

COLESTEROL-HDL MATERNO EN 94 MADRES EMBARAZADAS SANAS PROVENIENTES DE CINCO HOSPITALES EN TRES REGIONES DE CHILE, CON VALORES EXPRESADOS TANTO EN MG/DL COMO EN MMOL/L

\begin{tabular}{cccccccccc}
\hline Se & $n$ & $\begin{array}{c}\text { HDL-C } \\
\text { media } \\
\text { mg/dl }\end{array}$ & $\begin{array}{c}\text { HDL-C } \\
\text { DE } \\
\mathrm{mg} / \mathrm{dl}\end{array}$ & $\begin{array}{c}\text { HDL-C } \\
\mathrm{P}-90 \\
\mathrm{mg} / \mathrm{dl}\end{array}$ & $\begin{array}{c}\text { HDL-C } \\
\mathrm{P}-10 \\
\mathrm{mg} / \mathrm{dl}\end{array}$ & $\begin{array}{c}\text { HDL-C } \\
\text { media } \\
\mathrm{mmol} / \mathrm{l}\end{array}$ & $\begin{array}{c}\text { HDL-C } \\
\mathrm{DE} \\
\mathrm{mmol} / \mathrm{l}\end{array}$ & $\begin{array}{c}\text { HDL-C } \\
\mathrm{P}-90 \\
\mathrm{mmol} / \mathrm{l}\end{array}$ & $\begin{array}{c}\text { HDL-C } \\
\mathrm{P}-10 \\
\mathrm{mmol} / \mathrm{l}\end{array}$ \\
\hline 9 & 9 & 57,40 & 19,81 & 82,80 & 32,00 & 1,48 & 0,51 & 2,14 & 0,83 \\
11 & 15 & 63,11 & 13,41 & 80,30 & 45,92 & 1,63 & 0,35 & 2,08 & 1,19 \\
13 & 9 & 58,00 & 13,72 & 75,59 & 40,41 & 1,50 & 0,35 & 1,95 & 1,05 \\
22 & 21 & 72,85 & 7,28 & 82,18 & 63,52 & 1,88 & 0,19 & 2,13 & 1,64 \\
24 & 8 & 74,04 & 16,01 & 94,56 & 53,52 & 1,91 & 0,41 & 2,45 & 1,38 \\
27 & 6 & 69,00 & 21,50 & 96,56 & 41,44 & 1,78 & 0,56 & 2,50 & 1,07 \\
33 & 19 & 70,85 & 15,26 & 90,41 & 51,29 & 1,83 & 0,39 & 2,34 & 1,33 \\
35 & 4 & 75,00 & 8,36 & 85,72 & 64,28 & 1,94 & 0,22 & 2,22 & 1,66 \\
38 & 3 & 86,66 & 21,77 & 114,57 & 58,75 & 2,24 & 0,56 & 2,96 & 1,52 \\
\hline
\end{tabular}

$\mathrm{Se}=$ semanas de embarazo. $\mathrm{n}=$ número de pacientes. $\mathrm{HDL}-\mathrm{C}=$ Colesterol-HDL materno. Media=media aritmética (percentil 50). $\mathrm{DE}=$ desviación estándar. $\mathrm{P}-90=$ percentil 90 (=media + [1,282 x DE]). P-10=percentil 10 (= media - [1,282 x DE]).

Tabla IX

COLESTEROL-HDL MATERNO: ECUACIONES DE REGRESIÓN POLINOMIAL DE SEGUNDO ORDEN PARA LOS PERCENTILES (50, 90 Y 10), QUE PERMITIERON CONSTRUIR EL GRÁFICO DE LA FIGURA 5 CON VALORES EXPRESADOS TANTO EN MG/DL COMO EN MMOL/L

\begin{tabular}{llr}
\hline & Ecuación Polinomial de segundo orden & ${\text { Ecuación } \mathrm{N}^{\circ}}$ \\
\hline Percentil $50(\mathrm{mg} / \mathrm{dL})$ & $\mathrm{Y}=52,42+(0,6989 \mathrm{X})+\left(0,001181 \mathrm{X}^{2}\right)$ & 19 \\
Percentil $90(\mathrm{mg} / \mathrm{dL})$ & $\mathrm{Y}=81,75-(0,4002 \mathrm{X})+\left(0,02565 \mathrm{X}^{2}\right)$ & 20 \\
Percentil $10(\mathrm{mg} / \mathrm{dL})$ & $\mathrm{Y}=23,09+(1,798 \mathrm{X})-\left(0,02329 \mathrm{X}^{2}\right)$ & 21 \\
Percentil $50(\mathrm{mmol} / \mathrm{l})$ & $\mathrm{Y}=1,357+(0,01758 \mathrm{X})+\left(\left[4,241 \times 10^{-5}\right] \mathrm{X}^{2}\right)$ & 22 \\
Percentil $90(\mathrm{mmol} / \mathrm{l})$ & $\mathrm{Y}=2,103-(0,009126 \mathrm{X})+\left(0,0006379 \mathrm{X}^{2}\right)$ & 23 \\
Percentil $10(\mathrm{mmol} / \mathrm{l})$ & $\mathrm{Y}=0,6090+(0,04543 \mathrm{X})-\left(0,0005815 \mathrm{X}^{2}\right)$ & 24 \\
\hline
\end{tabular}

$\mathrm{Y}=\mathrm{HDL}-\mathrm{C}$ (en $\mathrm{mg} / \mathrm{dL}$ para ecuaciones $19,20,21 \mathrm{y}$ en $\mathrm{mmol} / \mathrm{L}$ para las ecuaciones $22,23,24) . \mathrm{X}=$ semana de embarazo. 


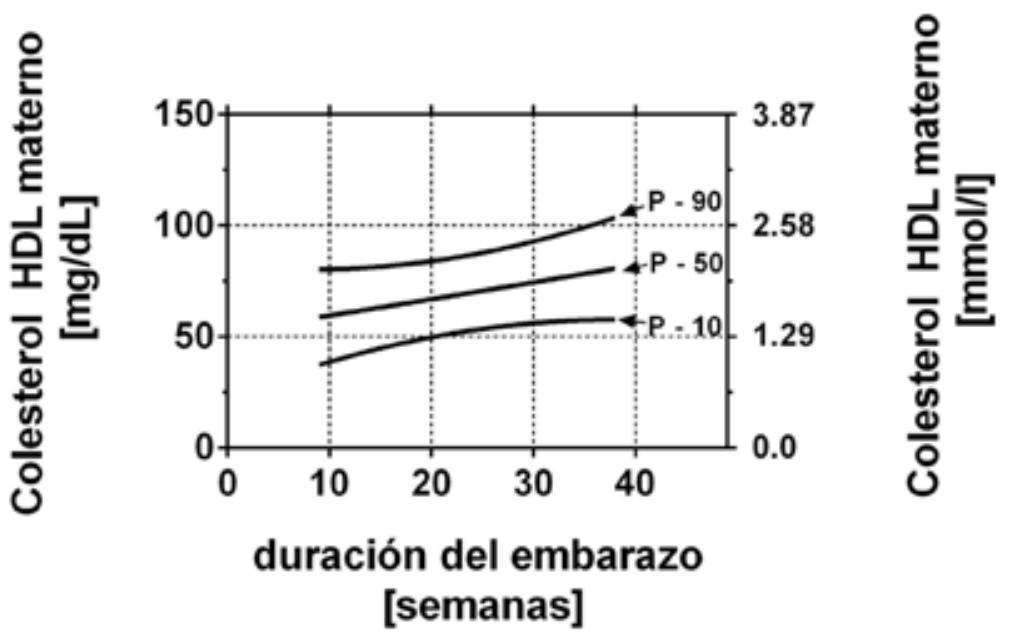

Figura 5. Colesterol HDL en el suero materno durante el embarazo. Percentil 50 (P-50), Percentil 90 (P-90) y Percentil 10 (P-10) calculados a partir de mujeres embarazadas sanas chilenas con IMC normal pregestacional. Valores de Colesterol HDL expresados tanto en $\mathrm{mg} / \mathrm{dL}$ (eje $\mathrm{Y}$, a izquierda) como en $\mathrm{mmol} / \mathrm{l}$ (eje $\mathrm{Y}$, a derecha).

Tabla X

ÁCIDOS GRASOS NO ESTERIFICADOS (NEFA) MATERNOS EN 94 EMBARAZADAS SANAS PROVENIENTES DE CINCO HOSPITALES DE TRES REGIONES DE CHILE, CON VALORES EXPRESADOS SOLAMENTE EN MMOL/L

\begin{tabular}{cccccc}
\hline Se & $\mathrm{n}$ & $\begin{array}{c}\text { NEFA } \\
\text { Media } \\
\mathrm{mmol} / \mathrm{l}\end{array}$ & $\begin{array}{c}\text { NEFA } \\
\text { DE } \\
\mathrm{mmol} / \mathrm{l}\end{array}$ & $\begin{array}{c}\text { NEFA } \\
\text { P-90 } \\
\mathrm{mmol} / \mathrm{l}\end{array}$ & $\begin{array}{c}\text { NEFA } \\
\mathrm{P}-10 \\
\mathrm{mmol} / \mathrm{l}\end{array}$ \\
\hline 9 & & & & \\
11 & - & 0,5720 & 0,1642 & 0,7825 & 0,3615 \\
13 & 15 & 0,5413 & 0,2234 & 0,8277 & 0,2549 \\
22 & 9 & 0,6371 & 0,2819 & 0,9985 & 0,2757 \\
24 & 21 & 0,6655 & 0,2928 & 1,0409 & 0,2901 \\
27 & 8 & & & & 0,9508 \\
33 & - & 0,6508 & 0,2340 & 1,1443 & 0,3508 \\
35 & 19 & 0,8375 & 0,2393 & & 0,5307 \\
38 & 4 & & & &
\end{tabular}

$\mathrm{Se}=$ semanas de embarazo. $\mathrm{n}=$ número de pacientes. NEFA= ácidos grasos no esterificados (ácidos grasos libres) maternos. Media=media aritmética (percentil 50). DE=desviación estándar. P-90=percentil $90(=$ media $+[1,282 \times \mathrm{DE}])$. P$10=$ percentil $10(=$ media $-[1,282 \times D E])$.

Tabla XI

ÁCIDOS GRASOS NO ESTERIFICADOS (NEFA) MATERNOS: ECUACIONES DE REGRESIÓN POLINOMIAL DE SEGUNDO ORDEN PARA LOS PERCENTILES (50, $90 \mathrm{Y}$ 10), QUE PERMITIERON CONSTRUIR EL GRÁFICO DE LA FIGURA 6 CON VALORES EXPRESADOS SOLAMENTE EN MMOL/L

\begin{tabular}{llc}
\hline & Ecuación Polinomial de segundo orden & Ecuación N $^{\circ}$ \\
\hline Percentil $50(\mathrm{mmol} / \mathrm{l})$ & $\mathrm{Y}=0,5451-(0,001147 \mathrm{X})+\left(0,0002161 \mathrm{X}^{2}\right)$ & 25 \\
Percentil $90(\mathrm{mmol} / \mathrm{l})$ & $\mathrm{Y}=0,4478+(0,03676 \mathrm{X})-\left(0,00055 \mathrm{X}^{2}\right)$ & 26 \\
Percentil 10 $(\mathrm{mmol} / \mathrm{l})$ & $\mathrm{Y}=0,6424-(0,03905 \mathrm{X})+\left(0,0009823 \mathrm{X}^{2}\right)$ & 27 \\
\hline
\end{tabular}

Y=ácidos grasos no esterificados (NEFA) maternos (expresados solo en mmol/l). X=semana de embarazo 

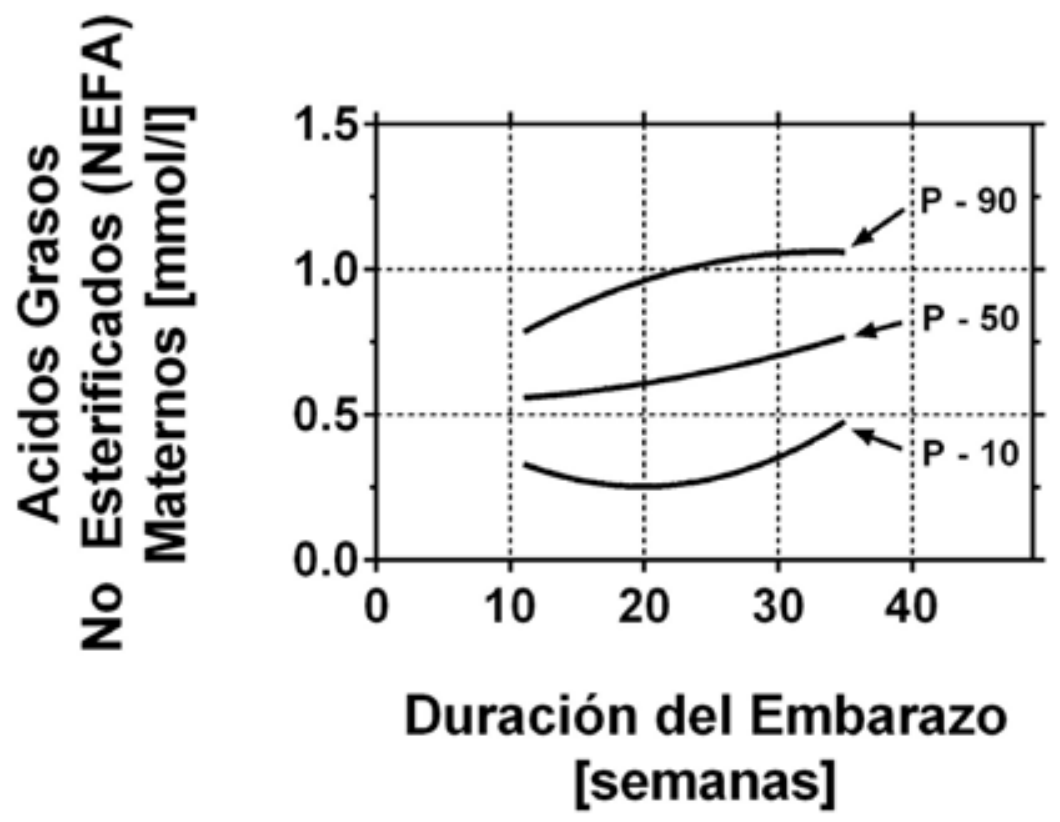

Figura 6. Ácidos grasos no esterificados (NEFA por sus siglas en inglés, o ácidos grasos libres) en el suero materno durante el embarazo. Percentil 50 (P-50), Percentil 90 (P-90) y Percentil 10 (P-10) calculados a partir de mujeres embarazadas sanas chilenas con IMC normal pregestacional. Valores de ácidos grasos no esterificados expresados solamente en $\mathrm{mmol} / \mathrm{l}$ (eje $\mathrm{Y}$, a izquierda).

\section{DISCUSIÓN}

Con respecto al nivel de colesterol total materno en el embarazo normal, se aceptaba en 2008 que en el momento del parto, éste ha aumentado $25-50 \%$ con respecto a su nivel pregestacional $(9,10)$. En nuestras pacientes el colesterol medio aumentó un $35,9 \%$ entre las 9 y 38 semanas.

El colesterol es captado por el trofoblasto placentario en forma de lipoproteínas que son internalizadas en forma tanto receptor-dependiente como receptor-independiente. En la primera mitad del embarazo, la concentración fetal de colesterol se correlaciona estrictamente con los niveles maternos, pero esta relación se pierde en el tercer trimestre, cuando el colesterol plasmático fetal disminuye (11).

En general se acepta que los triglicéridos maternos aumentan tres o cuatro veces durante el embarazo normal con respecto a sus valores pregestacionales (9). En nuestras pacientes el valor medio de triglicéridos aumentó 3,4 veces entre la semana 9 y la 38.

Fisiológicamente, el transporte transplacentario de triglicéridos (Figura 1) se incrementa por la hipertrigliceridemia materna fisiológica del embarazo. Esta última condición se debe a dos mecanismos: disminución del catabolismo de las lipoproteínas de muy baja densidad (VLDL) maternas, y una lipolisis acelerada en el tejido adiposo. El catabolismo de las VLDL en los tejidos adiposo y muscular maternos está bloqueada parcialmente por la resistencia insulínica fisiológica del embarazo, que reduce la actividad de la lipasa lipoproteica (LPL). Por otro lado, la misma resistencia a la insulina incrementa el flujo de ácidos grasos no esterificados y glicerol hacia el hígado, determinando una mayor síntesis hepática materna de VLDL. El resultado de la hipertrigliceridemia materna es un flujo global incrementado de VLDL hacia la placenta (12-15). Allí, la lipasa lipoproteica placentaria, que está presente en la membrana vellosa materna del sincicio-trofoblasto, hidroliza las moléculas de triglicéridos de las VLDL, transformándolas en ácidos grasos libres y glicerol, los que a su vez son re-esterificados como triglicéridos en el citotrofoblasto. Finalmente, las células del endotelio capilar fetal vuelven a hidrolizar a los triglicéridos, y los ácidos grasos así generados son transportados por la alfa-fetoproteína ( $\mathrm{aFP}$ ) sanguínea hacia los tejidos fetales.

Se ha reportado que el colesterol-HDL sube en el embarazo normal aproximadamente en $30 \%$ en comparación con mujeres no embarazadas, producto de mayor síntesis estimulada por los estrógenos 
placentarios $(16,10)$. En nuestras pacientes el colesterol-HDL medio aumentó un $33,7 \%$ entre la semana 9 y la 38 .

Los ácidos grasos libres (NEFA) aumentan hasta un $47 \%$ a lo largo del embarazo en mujeres sanas, debido a una tasa mayor de lipolisis, particularmente en el tercer trimestre $(17,18,19)$. En nuestras pacientes, el nivel medio de ácidos grasos libres aumentó un 31,7\% entre la semana 11 y la 35. La mayor dispersión de datos ocurrió en el segundo trimestre.

Desde 1984 (20) y al menos hasta el año 2008 la elevación de lípidos maternos observada durante el embarazo aún en ausencia de diabetes gestacional o pregestacional se hacía llamar "Lipemia Gestacional", y solamente se le asignaba importancia como posible predictor de dislipidemia y riesgo cardiovascular en la madre y la descendencia, años después del parto (10). Fue precisamente a comienzos de la década de 1980 cuando se publicaron las primeras tablas de lípidos maternos normales según edad gestacional $(10,21)$, especificando el percentil 90 de colesterol total y triglicéridos, y el percentil 10 de colesterol HDL. En efecto, la razón por la que nosotros elegimos los percentiles 50, 90 y 10 para delimitar la normalidad fue precisamente para que nuestros resultados fuesen fácilmente comparables con los de autores previos.

\section{CONCLUSIÓN}

Hemos cumplido nuestro objetivo de llenar la necesidad de generar tablas y gráficos de percentiles normales de lípidos séricos maternos según edad gestacional en población embarazada sana y sin sobrepeso, con población del país obtenida de varios centros asistenciales en más de una región geográfica. En efecto, mediante la cooperación de cinco Servicios de Obstetricia y Ginecología de tres regiones del país, logramos calcular las tablas y gráficos de lípidos maternos normales a lo largo del embarazo, que sean aplicables a la población de embarazadas chilenas.

\section{REFERENCIAS}

1. Coustan DR. Gestational Diabetes. Chapter 35. In: NDDG-National Diabetes Data Group. National Institutes of Health. National Institute of Diabetes and Digestive and Kidney Diseases. NIH Publication № 95-1468, 1995.

2. Hoffert-Gilmartin A, Ural SA, Repke JT. Gestational Diabetes Mellitus. Rev Obstet Gynecol 2008;1(3):12934.

3. Olmos PR, Belmar CG. Diabetes y Embarazo. En: Pérez A, Donoso E. Obstetricia. $4^{\circ}$ Edición 2011. Editorial Mediterráneo Ltda. Santiago, Chile. pp 1019-52.

4. Zhang X, Decker A, Platt RW, Kramer MS. How big is too big? The perinatal consequences of macrosomia. Am J Obstet Gynecol 2008;198:517.e1-6.
5. Merzouk $\mathrm{H}$, Khan NA. Implications of lipids in macrosomia of diabetic pregnancy; Can n-3 polynsaturated fatty acids exert beneficial effects? Clin Sci (Lond) 2003;105:519-29.

6. Chahuan SP, Grobman WA, Gherman RA, Chahuan VB, Chang G, Magann EF, Hendrix NW. Suspicion and treatment of the macrosomic fetus: A review. Am J Obstet Gynecol 2005;193:332-34.

7. Olmos P, Borzone G, Olmos R, Valencia C, Bravo F, Hodgson MI, Belmar C, Poblete J, Escalona M, Gómez B. Gestational diabetes and prepregnancy overweight: possible factors involved in newborn macrosomia. J Obstet Gynaecol Res 2012;38:208-214

8. Olmos P, Martelo G, Reimer V, Rigotti A, Busso D, Belmar C, González R, Goldenberg D, Samith B, Santos JL, Escalona M, Quezada T, Faúndez J, Nicklitschek I. La hipótesis de Pedersen no es suficiente: otros nutrientes además de la glucosa explicarían macrosomía fetal en diabéticas gestacionales con sobrepeso y buen control glicémico. Rev Med Chil 2013;141:1441-8.

9. Knopp R, Bonet B, Zhu X-D. Lipid metabolism in pregnancy. In: Cowett R; Editor. Principles of PerinatalNeonatal Metabolism. New York: Springer-Verlag; 1998. pp 221-58.

10. Knopp RH, Paramsothy P. Management of Hyper/ Dyslipidemias. En: Part 2. Management of Diabetic/ Medical Complications in Pregnancy. En: Kitzmiller JL, Jovanovic L, Brown F, Coustan D, Reader DM, (Eds). Managing preexisting diabetes and pregnancy. Technical reviews and consensus recommendations for care. (c) 2008 by The Americal Diabetes Association, Inc., 1701 North Beauregard Street, Alexandria, Virginia, USA, VI 22311. Pp 355-99.

11. Schmid KE, Davidson WS, Myatt L, Woolett LA. The transport of cholesterol across a placental cell monolayer: Implications for net transport of sterol from the maternal to the fetal circulation. J Lipid Res 2003;44:1909-18.

12. Shaefer-Graf UM, Graf K, Kulbancka I, Jjos SL, Dudenhausen J, Vetter K, Herrera E. Maternal lipids as strong determinants of fetal environment and growth in pregnancies with gestational diabetes mellitus. Diabetes Care 2008;31:1858-63.

13. Herrera E, Amusquivar E, López-Soldado I, Ortega $\mathrm{H}$. Maternal lipid metabolism and placental lipid transfer. Horm Res 2006;65 [Suppl. 3]: 59-64.

14. Magnusson-Olsson AL, Hamark B, Ericsson A, Wennergren M, Jansson T, Powell TL. Gestational and hormonal regulation of human placental lipoprotein lipase. J Lipid Res 2006;47:2551-61.

15. Zawiejska A, Wender-Ozegowska E, Brazert J, Sodowski K. Components of metabolic syndrome and their impact on fetal growth in women with gestational diabetes mellitus. J Physiol Pharmacol 2008;59 Suppl.4:5-18.

16. De J, Mukhopadhyay AK, Saha PK. Study of serum lipid profile in pregnancy induced hypertension. Indian J Clin Biochem 2006;21:165-8.

17. Alvino G, Cozzi V, Radaelli T, Ortega H, Herrera E, Cetin I. Maternal and fetal fatty acid profile in normal and intrauterine growth restriction pregnancies with and without preeclampsia. Pediatr Res 2008;64:615-20.

18. Herrera E, Ortega H, Alvino G, Giovannini N, Amusquivar $\mathrm{E}$, Cetin I. Relationship between plasma fatty 
acid profile and antioxidant vitamins during normal pregnancy. Eur J Clin Nutr 2004;58:1231-8.

19. Diderholm B, Strisdberg M, Ewald U, Lindeberg-Norden S, Gustafsson J. Increased lipolysis in non-obese pregnant woman studied in the third trimester. BJOG 2005;112:713-8.

20. Montes A, Walden CE, Knopp RH, Cheung M, Chapman MB, Albers JJ. Physiologic and supraphysiologic increases in lipoprotein lipids and apoproteins in late pregnancy and postpartum. Possible markers for the diagnosis of "prelipemia". Atherosclerosis 1984;4:40717.

21. Knopp RH, Chapman M, Bergelin R, Wahl PW, Warth MR, Irvine S. Relationships of lipoprotein lipids to mild fasting hyperglycemia and diabetes in pregnancy. Diabetes Care 1980;3:416-20. 